\title{
We really need a classification of prostatic abscess?
}

\author{
KEY WORDS: Prostatic abscess; Needle aspiration; Classification.
}

Dear Editors,

I read with interest the article by the esteemed collegue Dell'Atti (1). However, I would provide some questions about the topic. Prostatic abscesses (PA) are now no longer a rare occurrence in clinical diagnostic urological practice. The wide use of antibiotics in patients with lower urinary tract symptoms (LUTS) and the high number of performed prostate biopsies could be responsible of the growing PA incidence in the last years (2). Several are the cases reports or the short series published in the scientific literature, as recently I have done with my clinical group in this same journal (3). I agree with Dell'Atti about the lack of a standardized diagnostic and therapeutic routines but I disagree about the excessive use of sub-classification as part of the five types of prostatic abscesses recognized (1). This classification seems to start from a pioneering work presented some years ago by Galosi et al. (4), in which the authors sought primarily to provide a tool for rapid employment to all those engaged to perform a prostatic ultrasound examination for routinary urological or andrological indications. I think that in case of PA the goal should be different. A classification should be characterized by morphological and clinical correlations but should allow an immediate approach since it is an urgent situation. What the urologist must be take into account is that the routine use of transrectal ultrasound (TRUS) evaluation in each case of LUTS associated to fever or predisposing factors for the development of PA may aid to avoid poor clinical evolutions of infection (5). In my opinion, the "Dell'Atti classification" does not reveal the exact correlation between the type of abscess and the type of treatment. In an urgent situation as the presence of a symptomatic prostatic abscess, which parameter should drive the therapeutic decision making? Are the dimensions crucial? Location into or around the gland may lead to an invasive or conservative therapeutic approach? I tried to apply the "Dell'Atti classification" in my local short series of prostatic abscesses realizing the impossibility of using the classes 1 and 3. From a prognostic point of view, only the PA dimensions and the associated symptoms had an extreme importance.

As showed in Table 1 (in the Supplementary Materials posted in www.aiua.it), in the last 30 months I prospectively collected 13 cases of symptomatic PA. Independently from the abscess diameter, all cases were treated by a TRUS-guided needle aspiration as first approach. In one case, with extensive involvement of seminal vescicles, it was necessary to perform a radical cistoprostatectomy with urinary diversion due to the inefficient result of needle ultrasound guided aspiration regarding the septic status. Recurrence were observed in four cases, which were managed by a second look of TRUS-guided needle aspiration. In two of these cases, a surgical adenomectomy was performed due to the persistence of bladder outlet obstruction. In this short series, the majors determinant factors of a poor prognosis were the PA dimensions and the seminal vesicles involvement. I believe that the approach of needle aspiration in the first place is essential for pathogenic diagnosis and then for the initial therapy. In the absence of symptoms, if a lesion is sonographically identified as PA, this lesion is only a cyst until proven otherwise, but because of the lack of symptoms the lesion should not be treated. What we need is a model of therapeutic management of the PA that allows us to treat it quickly and as best we can. In fact, we always keep in mind that PA is a potential life threatening condition that, when not adequately treated, may progress to sepsis and death. Thus, an accurate and rapid diagnostic work up and an efficient treatment are both required. As suggested by Vias et al. (5), a dimensional cut-off of $2 \mathrm{~cm}$ may aid to make a prompt therapeutic decision. In addition it is considered that the PA multilocularity could be an additional element of choice concerning the type of therapeutic approach considering that both transrectal needle aspiration and surgical approach (adenomectomy or transurethral deroofing) are viable comparable treatment option for PA (6). Needle aspiration, when done for properly selected cases (solitary lesion or multifocal but accessible via transrectal route), could minimize the morbidity of the drainage procedure (6).

\section{References}

1. Dell'Atti L. A new ultrasound and clinical classification for management of prostatic abscess. Arch Ital Urol Androl. 2015; 87:246-9.

2. Granados EA, Riley G, Salvador J, et al. Prostatic abscess: Diagnosis and treatment. J Urol. 1992; 148:80-2.

3. Fabiani A, Filosa A, Maurelli V, et al. Diagnostic and therapeutic utility of transrectal ultrasound in urological office prostatic abscess management: a short report from a single urologic center. Arch Ital Urol Androl. 2014; 86:344-8.

4. Galosi AB, Montironi R, Fabiani A, et al. Cystic Lesions of the Prostate Gland: An Ultrasound Classification With Pathological Correlation J Urol. 2009; 181:647-657.

5. Vias BJ, Ganpule SA, Ganpule AP, et al. Transrectal ultrasound-guided aspiration in the management of prostatic abscess: A single-center experience. Indian J Radiol Imaging. 2013; 23:253-257.

6. Elshal AM, Abdelhalim A, Barakat TS, et al. Prostatic abscess: Objective assessment of the treatment approach in the absenceof guidelines. Arab Journal of Urology. 2014; 12:262-268.

\section{Correspondence}

Andrea Fabiani, MD - andreadoc1@libero.it

Surgery Dpt, Section of Urology, ASUR Marche Area Vasta 3,

Macerata Hospital, Macerata, Italy
Andrea Fabiani Surgery Dpt, Section of Urology, ASUR Marche Area Vasta 3, Macerata Hospital, Macerata, Italy

No conflict of interest declared. 